\title{
Should genetic groups be fitted in BLUP evaluation? Practical answer for the French AI beef sire evaluation
}

\author{
Florence Phocas, Denis LaloË* \\ Station de génétique quantitative et appliquée, Institut national de la recherche agronomique, \\ 78352 Jouy-en-Josas Cedex, France
}

(Received 27 February 2003; accepted 29 December 2003)

\begin{abstract}
Some analytical and simulated criteria were used to determine whether a priori genetic differences among groups, which are not accounted for by the relationship matrix, ought to be fitted in models for genetic evaluation, depending on the data structure and the accuracy of the evaluation. These criteria were the mean square error of some extreme contrasts between animals, the true genetic superiority of animals selected across groups, i.e. the selection response, and the magnitude of selection bias (difference between true and predicted selection responses). The different statistical models studied considered either fixed or random genetic groups (based on six different years of birth) versus ignoring the genetic group effects in a sire model. Including fixed genetic groups led to an overestimation of selection response under BLUP selection across groups despite the unbiasedness of the estimation, i.e. despite the correct estimation of differences between genetic groups. This overestimation was extremely important in numerical applications which considered two kinds of within-station progeny test designs for French purebred beef cattle AI sire evaluation across years: the reference sire design and the repeater sire design. When assuming a priori genetic differences due to the existence of a genetic trend of around $20 \%$ of genetic standard deviation for a trait with $\mathrm{h}^{2}=0.4$, in a repeater sire design, the overestimation of the genetic superiority of bulls selected across groups varied from about $10 \%$ for an across-year selection rate $\mathrm{p}=1 / 6$ and an accurate selection index (100 progeny records per sire) to $75 \%$ for $\mathrm{p}=1 / 2$ and a less accurate selection index (20 progeny records per sire). This overestimation decreased when the genetic trend, the heritability of the trait, the accuracy of the evaluation or the connectedness of the design increased. Whatever the data design, a model of genetic evaluation without groups was preferred to a model with genetic groups when the genetic trend was in the range of likely values in cattle breeding programs ( 0 to $20 \%$ of genetic standard deviation). In such a case, including random groups was pointless and including
\end{abstract}

${ }^{*}$ Corresponding author: laloe@ dga.jouy.inra.fr 
fixed groups led to a large overestimation of selection response, smaller true selection response across groups and larger variance of estimation of the differences between groups. Although the genetic trend was correctly predicted by a model fitting fixed genetic groups, important errors in predicting individual breeding values led to incorrect ranking of animals across groups and, consequently, led to lower selection response.

selection bias / accuracy / genetic trend / connection / beef cattle

\section{INTRODUCTION}

More and more often, genetic evaluations deal with heterogeneous populations, dispersed over time and space. The reference method to get an accurate and unbiased prediction of breeding values of animals with records made at different time periods and in different environments (herds, countries...) is the best linear unbiased prediction (BLUP) under a mixed model including all information and pedigree from a base population where animals with unknown parents are unselected and sampled from a normal distribution with a zero mean and a variance equal to twice the Mendelian variance [4]. Considering the breeding values of animals in a mixed model as random effects from a homogeneous distribution implies the assumption that the breeding values of base animals have the same expectation, whatever their age or their geographical origin. A violation of this assumption can lead to an underestimation of genetic trend and to a biased prediction of breeding values. Including all data and pedigree information upon which selection is based, is often impossible in the practical world. Including fixed genetic groups overcomes the assumption of equality of expectations of breeding values across space and time [6], but the way to distinguish between the environmental and genetic parts of performance across different environments is not obvious [12]. Laloë and Phocas [9] showed that as soon as there is some confounding between genetic and environmental effects, the prediction of genetic trend may be strongly regressed towards a zero value when the average reliability of the evaluation is not large enough in well connected data designs of beef cattle breeding programs. Including fixed genetic groups in the evaluation leads to an unbiased estimation of differences between these groups, but also leads to less accurate estimated breeding values. In order to decide whether or not genetic groups ought to be considered in sire evaluation, two criteria have been proposed: the level of accuracy of comparisons between sires within the same group and between two sires in different groups [2] and the mean square error (MSE) of differences between groups [7]. Kennedy [7] showed that, in terms of minimising MSE, 
an operational model that ignores genetic groups is preferable to a model that accounts for differences between genetic groups if the true difference between genetic groups is not large enough. He proved that ignoring genetic groups leads to smaller MSE of the genetic contrasts across groups than the PEV under a model with genetic groups, as soon as the true genetic difference is less than the standard error of estimation of this between group difference. However, the proof could not be extended over two groups. Kennedy's argument was related to the classical statistical problem about accuracy versus bias. A more practical argument will be based on the efficiency of selection (by truncation on the estimated breeding values) induced by the evaluation model. In this paper, both kinds of criteria will be used to decide whether or not groups should be included in a genetic evaluation.

The numerical application concerns two kinds of progeny test design for sire evaluation in French beef cattle breeds [9]. Although these designs are really specific to France, they are quite illustrative of the problem of connectedness met with any beef cattle genetic evaluation because of the practical limitations of semen exchanges in many beef cattle herds. Indeed, some confounding may often be encountered between herd-year effects and genetic values of some animals like natural service bulls used within a herd and year. In the French AI beef sire evaluation, most of the bulls have their progeny performance recorded within a single year and only a few connecting bulls had progeny in different years in order to ensure some genetic links across years. The genetic group definition is based on the year of birth of the sires, assuming that no pedigree and records for sires are available and the sires are sampled from a selected base population. The genetic groups will be included as either random or fixed effects in the statistical model. Usually, genetic groups are considered as fixed effects, but some authors (e.g. [3]) advocate treating genetic groups as random effects when small amounts of data and pedigree information are available. In our numerical application, sire relationships were ignored, because relationships are not numerous in the open breeding nuclei of the French beef cattle breeds. Moreover, accounting for relationships may confuse the issue and do not allow a clear interpretation, because the results may strongly vary according to the degree of the relationships [4, 8]. Pollak and Quaas [11] have explained that the grouping of base animals is the only relevant grouping and they have shown that differences between groups decrease as more information is included in the relationship matrix. Empirical evidence has shown, however, that the use of relationships between sires does not completely account for the large existing genetic differences between groups when migration occurs without tracing back the common ancestors of animals in different areas $[7,12]$. 
In this paper, we will not formally consider phantom parent grouping strategies [13] because relationships are not taken into account. However, ignoring relationships will not remove anything to the generality of our conclusions, since this paper deals with the problem of grouping of base animals.

The aim of this research was to answer the following question: does a model that includes groups lead to a more efficient ranking of animals across groups and consequently a higher selection response? Criteria based on the analytical derivation of the selection bias under a model including genetic groups and on empirical expectations of true and predicted responses to selection are developed to determine whether a priori differences among genetic groups ought to be included in genetic evaluation.

\section{METHODS}

\subsection{Models and notations}

Let us consider the following mixed model:

$$
\mathbf{y}=\mathbf{X b}+\mathbf{Z} \mathbf{u}+\mathbf{e}
$$

where: $\mathbf{y}$ is the vector of performances, $\mathbf{b}$ is the vector of fixed effects, $\mathbf{u}$ is the vector of random genetic effects and $\mathbf{e}$ is the residual. $\mathbf{X}$ and $\mathbf{Z}$ are the corresponding matrices of incidence.

$\mathbf{u}$ can concern either the animals whose performance $\mathbf{y}$ are recorded, or their sires; thus, the genetic model is either an animal model or a sire model.

The distribution of random factors is:

$$
\left(\begin{array}{l}
\mathbf{u} \\
\mathbf{e}
\end{array}\right) \sim \mathbf{N}\left[\left(\begin{array}{l}
\mathbf{0} \\
\mathbf{0}
\end{array}\right),\left(\begin{array}{cc}
\mathbf{A} \sigma_{u}^{2} & \mathbf{0} \\
\mathbf{0} & \mathbf{I} \sigma_{e}^{2}
\end{array}\right)\right] .
$$

In this model, BLUE of $\mathbf{b}$ and BLUP of $\mathbf{u}$ are solutions of [5]:

$$
\left(\begin{array}{cc}
\mathbf{X}^{\prime} \mathbf{X} & \mathbf{X}^{\prime} \mathbf{Z} \\
\mathbf{Z}^{\prime} \mathbf{X} & \mathbf{Z}^{\prime} \mathbf{Z}+\lambda \mathbf{A}^{-1}
\end{array}\right)\left(\begin{array}{c}
\hat{\mathbf{b}} \\
\hat{\mathbf{u}}
\end{array}\right)=\left(\begin{array}{c}
\mathbf{X}^{\prime} \mathbf{y} \\
\mathbf{Z}^{\prime} \mathbf{y}
\end{array}\right)
$$

where $\lambda$ is the ratio $\sigma_{e}^{2} / \sigma_{u}^{2}$.

The classical way of accounting for systematic genetic differences between animals is to introduce genetic groups in the model, i.e.:

$$
\mathbf{y}=\mathbf{X b}+\mathbf{Q g}+\mathbf{Z} \mathbf{u}+\mathbf{e}
$$

where: $\mathbf{y}$ is the vector of performance, $\mathbf{b}$ is the vector of the fixed effects, $\mathbf{g}$ is the vector of random (model II) or fixed (model III) effects of $n$ genetic 
groups, $\mathbf{e}$ is the residual vector, $\mathbf{u}$ is the vector of random effects of animals as a deviation from their group expectation. $\mathbf{X}, \mathbf{Q}$ and $\mathbf{Z}$ are the corresponding matrices of incidence.

BLUE (best linear unbiased estimator) of $\mathbf{b}$ (and $\mathbf{g}$ treated as a fixed effect) and BLUP of $\mathbf{u}$ (and $\mathbf{g}$ treated as a random effect) are solutions (e.g., [5]) of the equations system:

$$
\left(\begin{array}{ccc}
\mathbf{X}^{\prime} \mathbf{X} & \mathbf{X}^{\prime} \mathbf{Q} & \mathbf{X}^{\prime} \mathbf{Z} \\
\mathbf{Q}^{\prime} \mathbf{X} & \mathbf{Q}^{\prime} \mathbf{Q}+\eta \mathbf{I} & \mathbf{Q}^{\prime} \mathbf{Z} \\
\mathbf{Z}^{\prime} \mathbf{X} & \mathbf{Z}^{\prime} \mathbf{Q} & \mathbf{Z}^{\prime} \mathbf{Z}+\lambda \mathbf{A}^{-\mathbf{1}}
\end{array}\right)\left(\begin{array}{c}
\hat{\mathbf{b}} \\
\hat{\mathbf{g}} \\
\hat{\mathbf{u}}
\end{array}\right)=\left(\begin{array}{c}
\mathbf{X}^{\prime} \mathbf{y} \\
\mathbf{Q}^{\prime} \mathbf{y} \\
\mathbf{Z}^{\prime} \mathbf{y}
\end{array}\right) .
$$

If $\mathbf{g}$ is a random effect, $\eta=\sigma_{\mathrm{e}}^{2} / \sigma_{\mathrm{g}}^{2}$. If $\mathbf{g}$ is a fixed effect, $\eta \mathbf{I}$ is ignored.

\subsection{Prediction error variance (PEV) and mean square error (MSE) of genetic contrasts}

Under model I, the variance-covariance matrix of the errors of estimation of fixed effects and prediction errors of random effects (PEV), is written as:

$$
\operatorname{var}\left(\begin{array}{c}
\hat{\mathbf{b}} \\
\hat{\mathbf{u}}-\mathbf{u}
\end{array}\right)=\left(\begin{array}{cc}
\mathbf{X}^{\prime} \mathbf{X} & \mathbf{X}^{\prime} \mathbf{Z} \\
\mathbf{Z}^{\prime} \mathbf{X} & \mathbf{Z}^{\prime} \mathbf{Z}+\lambda \mathbf{A}^{-1}
\end{array}\right)^{-1} \sigma_{\mathrm{e}}^{2}
$$

The prediction error variance of a linear combination $\mathbf{x}^{\prime} \hat{\mathbf{u}}$ is derived as:

$$
\operatorname{PEV}\left(\mathbf{x}^{\prime} \hat{\mathbf{u}}\right)=\mathbf{x}^{\prime} \operatorname{var}(\hat{\mathbf{u}}-\mathbf{u}) \mathbf{x} \text {. }
$$

MSE are more relevant than PEV, in particular if systematic differences between animals are known to occur and $E(\mathbf{u})$ is not null, possibly leading to biased estimated breeding values. The MSE of prediction is the sum of the error variance of prediction (PEV) and the squared bias of prediction. If a predictor is unbiased, MSE and PEV are equal. If $\mathrm{E}(\mathbf{u})$ is a priori known, the bias $\mathrm{E}(\hat{\mathbf{u}} \mid \mathrm{E}(\mathbf{u}))$ can be computed by use of the formulae given in [9]. $\mathrm{d}_{\mathrm{x}^{\prime} \hat{\mathrm{u}}}^{2}$.

If we denote $\mathrm{d}_{\mathrm{x}^{\prime} \hat{\mathrm{u}}}$ the bias in $\mathbf{x}^{\prime} \hat{\mathbf{u}}$ under model I, $\operatorname{MSE}\left(\mathbf{x}^{\prime} \hat{\mathbf{u}}\right)=\mathbf{x}^{\prime} \operatorname{var}(\hat{\mathbf{u}}-\mathbf{u}) \mathbf{x}+$

With the Henderson notation [4], $\mathbf{x}^{\prime} \mathbf{u}$ becomes $\mathbf{L}^{\prime} \mathbf{u}$ and the type of selection concerned is called the " $\mathbf{L}^{\prime} \mathbf{u}$ selection", i.e. $\mathrm{E}\left(\mathbf{L}^{\prime} \mathbf{u}\right)=\mathrm{d}$ with $\mathrm{d}$ non equal to 0 . Henderson [4] defined that there is $\mathbf{L}^{\prime} \mathbf{u}$ selection when some knowledge of values of sires exists external to records to be used in the evaluation.

Under model II or model III, the variance-covariance matrice of estimation and prediction errors is written as:

$$
\operatorname{Var}\left(\begin{array}{c}
\hat{\mathbf{b}} \\
\hat{\mathbf{g}}-\mathbf{g} \\
\hat{\mathbf{u}}-\mathbf{u}
\end{array}\right)=\left(\begin{array}{ccc}
\mathbf{X}^{\prime} \mathbf{X} & \mathbf{X}^{\prime} \mathbf{Q} & \mathbf{X}^{\prime} \mathbf{Z} \\
\mathbf{Q}^{\prime} \mathbf{X} & \mathbf{Q}^{\prime} \mathbf{Q}+\eta \mathbf{I} & \mathbf{Q}^{\prime} \mathbf{Z} \\
\mathbf{Z}^{\prime} \mathbf{X} & \mathbf{Z}^{\prime} \mathbf{Q} & \mathbf{Z}^{\prime} \mathbf{Z}+\lambda \mathbf{A}^{-1}
\end{array}\right)^{-1} \sigma_{\mathrm{e}}^{2}
$$


Estimated breeding value $\hat{a}_{i j}$ of an animal $j$ belonging to the genetic group $i$ is expressed as $\hat{a}_{i j}=\hat{\mathrm{g}}_{i}+\hat{\mathrm{u}}_{i j}$ when $\mathrm{a}_{i j}=\mathrm{g}_{i}+\mathrm{u}_{i j}$ and $\mathrm{u}_{i j}$ and $\hat{\mathrm{u}}_{i j}$ are respectively the true and predicted genetic value of the animal $j$, expressed intra-group.

In the vectorial form, it can be written as: $\hat{\mathbf{a}}=\mathbf{K} \hat{\mathbf{g}}+\hat{\mathbf{u}}$, where $\mathbf{K}$ is a matrix with a number of rows equal to the number of animals and a number of columns equal to the number of groups. $\mathbf{K}(i, j)$ is equal to 1 if animal $j$ belongs to group $i, 0$ otherwise.

$$
\begin{aligned}
& \operatorname{var}(\hat{\mathbf{a}}-\mathbf{a})=\mathbf{K} \operatorname{var}(\hat{\mathbf{g}}-\mathbf{g}) \mathbf{K}^{\prime}+\operatorname{var}(\hat{\mathbf{u}}-\mathbf{u})+2 \mathbf{K} \operatorname{cov}(\hat{\mathbf{g}}-\mathbf{g}, \hat{\mathbf{u}}-\mathbf{u}) \\
& \operatorname{PEV}^{*}\left(\mathbf{x}^{\prime} \hat{\mathbf{a}}\right)=\mathbf{x}^{\prime} \operatorname{var}(\hat{\mathbf{a}}-\mathbf{a}) \mathbf{x} .
\end{aligned}
$$

If we denote $\mathrm{d}_{\mathrm{x}^{\prime}} \hat{\mathrm{a}}$ the bias in $\mathbf{x}^{\prime} \hat{\mathbf{a}}, \operatorname{MSE}{ }^{*}\left(\mathbf{x}^{\prime} \hat{\mathbf{a}}\right)=\mathbf{x}^{\prime} \operatorname{var}(\hat{\mathbf{a}}-\mathbf{a}) \mathbf{x}+\mathrm{d}_{\mathbf{x}^{\prime} \hat{\mathbf{a}}}^{2}$.

If $\mathbf{g}$ is treated as fixed, the bias in $\mathbf{x}^{\prime} \mathbf{a}$ is zero and MSE* reduces to PEV*.

\subsection{Expectation of selection bias across genetic groups}

Let us call $\mathrm{R}$ and $\hat{\mathrm{R}}$, respectively the true and predicted responses to selection when selecting across the $n$ groups a proportion $\mathrm{P}$ of animals in a population of size $N$, based on their estimated breeding values $\hat{\mathrm{g}}_{i}+\hat{\mathrm{u}}_{i l}$. Let $\mathrm{k}_{i}$ be the number of animals selected from group $i ; \mathrm{k}_{i}$ depends on the value $\hat{\mathrm{g}}_{i}$ and, consequently is not a constant when deriving the expectation of selection bias.

$$
\mathrm{R}=\frac{1}{N \mathrm{P}} \sum_{i=1}^{n} \mathrm{k}_{i}\left(\mathrm{~g}_{i}+\frac{1}{\mathrm{k}_{i}} \sum_{l=1}^{\mathrm{k}_{i}} \mathrm{u}_{i l}\right) \text { and } \hat{\mathrm{R}}=\frac{1}{N \mathrm{P}} \sum_{i=1}^{n} \mathrm{k}_{i}\left(\hat{\mathrm{g}}_{i}+\frac{1}{\mathrm{k}_{i}} \sum_{l=1}^{\mathrm{k}_{i}} \hat{\mathrm{u}}_{i l}\right) \text {. }
$$

$\mathrm{P}$ is the constant overall selection rate; $\mathrm{P}=\sum_{i=1}^{n} \mathrm{k}_{i} / N$.

$$
\begin{aligned}
& \mathrm{E}(\mathrm{R})=\frac{1}{N \mathrm{P}} \sum_{i=1}^{n}\left(\mathrm{E}\left(\mathrm{k}_{i} \mathrm{~g}_{i}\right)+\sum_{l=1}^{\mathrm{k}_{i}} \mathrm{E}\left(\mathrm{u}_{i l}\right)\right) . \\
& \mathrm{E}(\hat{\mathrm{R}})=\frac{1}{N \mathrm{P}} \sum_{i=1}^{n}\left(\mathrm{E}\left(\mathrm{k}_{i} \hat{\mathrm{g}}_{i}\right)+\sum_{l=1}^{\mathrm{k}_{i}} \mathrm{E}\left(\hat{\mathrm{u}}_{i}\right)\right) . \\
& \mathrm{E}\left(\mathrm{k}_{i} \mathrm{~g}_{i}\right)=\operatorname{cov}\left(\mathrm{k}_{i}, \mathrm{~g}_{i}\right)+\mathrm{E}\left(\mathrm{k}_{i}\right) \mathrm{E}\left(\mathrm{g}_{i}\right) . \\
& \mathrm{E}\left(\mathrm{k}_{i} \hat{\mathrm{g}}_{i}\right)=\operatorname{cov}\left(\mathrm{k}_{i}, \hat{\mathrm{g}}_{i}\right)+\mathrm{E}\left(\mathrm{k}_{i}\right) \mathrm{E}\left(\hat{\mathrm{g}}_{i}\right) .
\end{aligned}
$$

Due to the property of unbiasedness of BLUE and BLUP, $\mathrm{E}\left(\hat{\mathrm{g}}_{i}\right)=\mathrm{E}\left(\mathrm{g}_{i}\right)$ and $\mathrm{E}\left(\hat{\mathrm{u}}_{i l}\right)=\mathrm{E}\left(\mathrm{u}_{i l}\right)$. 
Consequently, the selection bias is written as:

$$
\mathrm{E}(\hat{\mathrm{R}}-\mathrm{R})=\frac{1}{N \mathrm{P}} \sum_{i=1}^{n}\left(\operatorname{cov}\left(\mathrm{k}_{i}, \hat{\mathrm{g}}_{i}-\mathrm{g}_{i}\right)\right)
$$

Under repeated sampling and for a given set of $\mathrm{g}_{i}, \mathrm{k}_{i}$ increases when $\hat{\mathrm{g}}_{i}-\mathrm{g}_{i}$ increases. To illustrate this point, let us imagine a case where there are not different subpopulations, i.e. $\mathrm{g}_{i}=0$ whatever $i$. However, the statistician believes that $\mathrm{g}_{i} \neq 0$ and, consequently, applies a statistical model including genetic groups as either random or fixed effects. For a given sample, the estimation of $\mathrm{g}_{i}$ leads to the under-estimation of some $\mathrm{g}_{i}$ and to the over-estimation of other $\mathrm{g}_{i}$, although the property $\mathrm{E}\left(\hat{\mathrm{g}}_{i}\right)=\mathrm{E}\left(\mathrm{g}_{i}\right)$ is respected. Because selection for the best EBV depends on the $\hat{\mathrm{g}}_{i}$, animals belonging to the overestimated groups are chosen to the detriment of animals belonging to the underestimated groups and $\hat{\mathrm{R}}$ is superior to $\mathrm{R}$ for a given sample. Under repeated sampling, $\hat{\mathrm{g}}_{i}$ may be ranked in different orders, but, in each sample, $\hat{\mathrm{R}}$ will be greater than $\mathrm{R}$ and, consequently, $\mathrm{E}(\hat{\mathrm{R}}-\mathrm{R})>0$ when there are not different subpopulations in reality.

Whatever the reality of the different subpopulations, $\operatorname{cov}\left(\mathrm{k}_{i}, \mathrm{~g}_{i}\right)=0$ when $\mathrm{g}_{i}$ are considered as fixed effects in the statistical model. In such a case, the selection bias is given by the following formula: $\mathrm{E}(\hat{\mathrm{R}}-\mathrm{R})=\frac{1}{N \mathrm{P}} \sum_{i=1}^{n}\left(\operatorname{cov}\left(\mathrm{k}_{i}, \hat{\mathrm{g}}_{i}\right)\right)$. When $\hat{\mathrm{g}}_{i}$ increases, $\mathrm{k}_{i}$ increases; then $\operatorname{cov}\left(\mathrm{k}_{i}, \hat{\mathrm{g}}_{i}\right)>0$ and $\mathrm{E}(\hat{\mathrm{R}})>\mathrm{E}(\mathrm{R})$.

The above formulae demonstrate that, in case of truncation selection based on EBV across groups, the expectation of the predicted response to selection $E(\hat{R})$ is greater than the expectation of the true response to selection $E(R)$ when $\mathrm{g}_{i}$ is considered as a fixed effect. The only necessary condition to obtain this result is to consider the unbiasedness properties of the best linear unbiased estimators and predictors (BLUE and BLUP) demonstrated by Henderson [5] under a model where random effects are specified correctly (e.g., Kennedy [7]).

\section{NUMERICAL APPLICATION}

The numerical application considers the two progeny test designs for French beef AI sire evaluation which were completely described in a previous paper of Laloë and Phocas [9]. This application was studied because of the questions arising from breeding selection units about the effect of the degree of connectedness across years on the efficiency of their selection program for AI bulls. 
The reference sire design

\begin{tabular}{|llllllll|}
\hline & Progeny number & \multicolumn{5}{c|}{ Number $(3+\mathrm{ns})$ of sires per year of evaluation $\mathrm{y}_{i}$} \\
& per sire and year & $\mathrm{y}_{1}$ & $\mathrm{y}_{2}$ & $\mathrm{y}_{3}$ & $\mathrm{y}_{4}$ & $\mathrm{y}_{5}$ & $\mathrm{y}_{6}$ \\
\hline Reference sires & $\mathrm{np}=20$ & $3 \mathrm{~S}$ & $3 \mathrm{~S}$ & $3 \mathrm{~S}$ & $3 \mathrm{~S}$ & $3 \mathrm{~S}$ & $3 \mathrm{~S}$ \\
Other sires & $\mathrm{np}=20$ & $20 \mathrm{~S}_{1}$ & $20 \mathrm{~S}_{2}$ & $20 \mathrm{~S}_{3}$ & $20 \mathrm{~S}_{4}$ & $20 \mathrm{~S}_{5}$ & $20 \mathrm{~S}_{6}$ \\
\hline
\end{tabular}

The repeater sire design

\begin{tabular}{|llllllll|}
\hline & Progeny number & \multicolumn{5}{c|}{ Number $(\mathrm{ns} / 2+\mathrm{ns})$ of sires per year of evaluation $\mathrm{y}_{i}$} \\
& per sire and year & $\mathrm{y}_{1}$ & $\mathrm{y}_{2}$ & $\mathrm{y}_{3}$ & $\mathrm{y}_{4}$ & $\mathrm{y}_{5}$ & $\mathrm{y}_{6}$ \\
\hline Repeater sires & $\mathrm{np} / 2=10$ & $4 \mathrm{~S}_{0}+$ & $4 \mathrm{~S}_{1}+$ & $4 \mathrm{~S}_{2}+$ & $4 \mathrm{~S}_{3}+$ & $4 \mathrm{~S}_{4}+$ & $4 \mathrm{~S}_{5}+$ \\
& & $4 \mathrm{~S}_{1}$ & $4 \mathrm{~S}_{2}$ & $4 \mathrm{~S}_{3}$ & $4 \mathrm{~S}_{4}$ & $4 \mathrm{~S}_{5}$ & $4 \mathrm{~S}_{6}$ \\
\hline Other sires & $\mathrm{np}=20$ & $16 \mathrm{~S}_{1}$ & $16 \mathrm{~S}_{2}$ & $16 \mathrm{~S}_{3}$ & $16 \mathrm{~S}_{4}$ & $16 \mathrm{~S}_{5}$ & $16 \mathrm{~S}_{6}$ \\
\hline
\end{tabular}

$\mathrm{y}_{i}$ : year of evaluation; $\mathrm{S}$ : reference sires born in year $-\mathrm{L} ; \mathrm{S}_{i}$ : Sires born in year $i-\mathrm{L}$, where $\mathrm{L}$ is the sire age at the beginning of its evaluation. np: number of progeny recorded per sire, within a year $\mathrm{y}_{i}($ default $=20$, other value $=100)$; ns: number of sires, candidates for selection within a year $\mathrm{y}_{i}($ default $=20)$.

Figure 1. The reference sire design. The repeater sire design.

\subsection{Test scenarios}

Each year, some yearling sires are selected on the basis of their estimated breeding values from station performance testing [10]. Each year, progeny of yearling sires pre-selected on performance testing are grouped together in a station where recording of performance is done either on beef traits for male progeny or on breeding traits for female progeny. The sires are progeny-tested according to planned designs in order to ensure genetic links between years. Two kinds of design coexist at present in France: the "reference sire design" and the "repeater sire design" (see Fig. 1). In the reference sire design, the same three bulls have progeny across all years to ensure genetic links and they are not candidates for selection. On the contrary, the repeater sires have progeny over 2 consecutives years to ensure genetic links and belong to the group of candidates for selection within their second year of evaluation. It must be clear that without these planned connections, there will be a perfect confounding between the sire's year of birth and the year of evaluation.

\subsection{Simulation}

\subsubsection{Selection process}

Details and figures about the two designs are shown in Figure 1. For each design, ns (equal to 20) candidates for selection per year were considered; 
for each of them, np (equal to 20 or 100) progeny performance were recorded, respectively. For both designs, six years of evaluation were considered. An increasing expectation of sire breeding value per birth year $\Delta \mathrm{G}$ of $0,0.1 \sigma_{\mathrm{a}}, 0.2 \sigma_{\mathrm{a}}$ and $0.3 \sigma_{\mathrm{a}}$, respectively, was assumed, corresponding to the genetic trend that is not accounted for in the data structure used for the genetic evaluation, because candidates for selection were chosen each year out of a large population of calves selected for birth conditions and weaning traits.

The selection procedure of sires was in two steps:

(1) a within-year selection step with a 50\% selection rate among the ns young candidates ranked on their EBV in order to get the AI official access permission,

(2) an across-year selection step with a $\mathrm{P}$ selection rate $(\mathrm{P}=1 / 6$ or $1 / 2)$ out of the population of AI sires selected within each of the 6 years. This second step corresponds to the real use of proven sires across the nucleus and commercial herds.

\subsubsection{Monte-Carlo simulation description}

For Monte-Carlo simulations, breeding values (BV) of reference sires were sampled from a distribution $N\left(0, \sigma_{\mathrm{a}}^{2}\right)$. Breeding values of sires born in year $j$ were sampled from the distribution $N\left(\mathrm{~g}_{j}, \sigma_{\mathrm{a}}^{2}\right)$, where $\mathrm{g}_{j}=j \Delta \mathrm{G}$. For the sires progeny-tested within a unique year, expectations of the sire random effects are related to the year of their evaluation, while the expectations of reference sire effects are equal to 0 and the expectations of repeater sire effects are related to the year of their first evaluation. Traits were only recorded on progeny bred by unrelated sires and unknown dams. Arguments for such a simplification are detailed in [9]. Consequently, phenotypes $\mathbf{y}$ of progeny were simulated by adding their genotype (sire effect + sampling component $N\left(0.3 / 4 \sigma_{\mathrm{a}}^{2}\right)$ due to the dam effect and the Mendelian sampling) to an environmental random residual sampled from $N\left(0, \sigma_{\mathrm{e}}^{2}\right)$. The phenotypic variance $\left(\sigma_{\mathrm{p}}^{2}=\sigma_{\mathrm{a}}^{2}+\sigma_{\mathrm{e}}^{2}\right)$ was supposed to be 100 and two different heritabilities $\left(\mathrm{h}^{2}=\sigma_{\mathrm{a}}^{2} / \sigma_{\mathrm{p}}^{2}\right)$ were simulated: $\mathrm{h}^{2}=0.20$ or $\mathrm{h}^{2}=0.40$.

\subsubsection{Genetic evaluation}

The genetic evaluation was implemented under the three statistical models (I, II and III) defined in Section 2.1, where the vector of fixed effects concerned 
the evaluation years and the vector of random genetic effects was the sire effects. For models II and III, the genetic group effects were also fitted, either treated as random (II) or as fixed (III) effects.

Estimated breeding values (EBV) were derived simultaneously with the estimation of the variance components under the three models.

\subsection{Criteria for model comparison}

\subsubsection{Selection bias}

Selection response was measured as the genetic superiority of the sires selected on EBV over the average genetic level of candidates for selection. In the numerical default case, the (true and predicted) selection responses were derived as the average $\mathrm{BV}$ or EBV of the 10 best sires ranked on EBV compared to the average $\mathrm{BV}$ or $\mathrm{EBV}$ of the 120 candidates for selection evaluated across a 6-year period.

Two criteria of robustness of the selection process were then studied: the magnitude of the selection bias $\mathrm{E}(\hat{\mathrm{R}}-\mathrm{R}) / \mathrm{E}(\mathrm{R})$ and the expectation of the true selection response $E(R)$ over 2500 replicates of Monte-Carlo simulations in the default case $\left(\mathrm{h}^{2}=0.4\right.$ and $\left.\Delta \mathrm{G}=0.2 \sigma_{\mathrm{a}}\right)$ and over 1000 replicates in the other cases in order to reduce the computing cost.

\subsubsection{Mean square error of prediction of genetic difference between animals}

Kennedy [7] proposed on the basis of a single two groups derivation, MSE of the contrast between genetic values of animals across groups in order to decide whether or not genetic groups ought to be included in a sire model. Here, we will broaden this approach to more than two groups by computing PEV and MSE of different contrasts between genetic values of animals belonging to different groups. These criteria were computed by simulation under the different models I, II and III. In particular, differences between the two youngest cohorts (numbered 5 and 6) and between the two extreme cohorts (the oldest and the youngest ones) will be studied in our numerical applications: MSE $5-6$ and $\mathrm{MSE}_{1-6}$, respectively. 


\section{RESULTS}

\subsection{Overall effect of the inclusion of genetic groups on the criteria for model comparison}

Table I presents the expectation over 2500 replicates of the true selection response and the selection bias occurring when an annual genetic trend of $0.2 \sigma_{\mathrm{a}}$ for a trait of $\mathrm{h}^{2}=0.4$, cannot be accounted for by the data and pedigree information. Table II presents the corresponding expectations over 1000 replicates with $\mathrm{h}^{2}=0.2$.

MSE between extreme cohorts (numbered 1 and 6) always converged towards the same conclusion "random groups should be included in sire evaluation" whereas true selection response and MSE between the two youngest cohorts (numbered 5 and 6 ) favoured the model without genetic groups due to similar true selection responses and lower $\mathrm{MSE}_{5-6}$. This point illustrates that the conclusion about the best model depends on the criterion used.

When genetic groups were included in the statistical model, the overestimation of the selection response was extremely large (5 to 121\%) if the groups were treated as a fixed effect and it was moderate (0 to 24\%) if the groups were treated as a random effect. These simulation results confirmed the analytical formulae derived in Section 2.3. The true selection responses were very similar when no or random groups were considered in the evaluation model. When fixed genetic groups were included, the true selection response was lower (from 2 to $20 \%$ of the response without groups) if there was little information recorded per sire $(\mathrm{np}=20)$ and was slightly increased $(5 \%$ maximum) if the amount of information per sire was important $(n p=100)$. These results were explained by looking at the distributions of selected sires across years (next section).

\subsection{Distribution of selected sires across years}

For a genetic trend of $0.2 \sigma_{\mathrm{a}}$ for a trait of $\mathrm{h}^{2}=0.4$, Table III gives the number of AI sires selected within each of the six years of evaluation, in a repeater sire design. When genetic groups were ignored, almost the same number of sires was selected from each year because the EBV of sires were strongly regressed towards the same mean across age cohorts. On the contrary, more sires were selected in the youngest versus the oldest cohorts when genetic trend was taken into account in the evaluation model by fitting fixed genetic groups. The sire evaluation with random genetic groups gave a distribution of sires selected 
Table I. Criteria derived from the genetic evaluation model, for varying number (np) of progeny per sire and selection rate (P), for a trait of heritability $\mathrm{h}^{2}=0.40$, with an annual genetic trend $\Delta \mathrm{G}=0.2 \sigma_{\mathrm{a}}$.

\begin{tabular}{lcccccccc}
\hline & \multicolumn{7}{c}{ Repeater sire design } \\
\hline & $\mathrm{R}$ & $(\hat{\mathrm{R}}-\mathrm{R}) / \mathrm{R}$ & $\mathrm{MSE}_{5-6}$ & $\mathrm{MSE}_{1-6}$ & $\mathrm{R}$ & $(\hat{\mathrm{R}}-\mathrm{R}) / \mathrm{R}$ & $\mathrm{MSE}_{5-6}$ & $\mathrm{MSE}_{1-6}$ \\
\hline & & \multicolumn{7}{c}{ Statistical model ignoring genetic groups } \\
$\mathrm{np}=20 \mathrm{P}=1 / 6$ & $5.75(0.027)$ & -0.04 & 4.1 & 26.7 & $5.39(0.028)$ & -0.01 & 5.1 & 42.6 \\
$\mathrm{np}=100 \mathrm{P}=1 / 6$ & $6.88(0.021)$ & -0.03 & 1.8 & 6.5 & $6.36(0.022)$ & -0.02 & 2.7 & 35.8 \\
$\mathrm{np}=20 \mathrm{P}=1 / 2$ & $2.75(0.012)$ & -0.06 & 4.1 & 26.7 & $2.52(0.012)$ & -0.01 & 5.1 & 42.6 \\
\hline & & \multicolumn{7}{c}{ Statistical model including random genetic groups } \\
$\mathrm{np}=20 \mathrm{P}=1 / 6$ & $5.81(0.028)$ & +0.12 & 9.1 & 11.7 & $5.37(0.028)$ & +0.02 & 5.9 & 41.1 \\
$\mathrm{np}=100 \mathrm{P}=1 / 6$ & $6.92(0.021)$ & +0.03 & 2.4 & 2.5 & $6.42(0.022)$ & -0.01 & 2.8 & 31.7 \\
$\mathrm{np}=20 \mathrm{P}=1 / 2$ & $2.82(0.013)$ & +0.16 & 9.1 & 11.7 & $2.51(0.012)$ & +0.02 & 5.9 & 41.1 \\
\hline & & \multicolumn{7}{c}{ Statistical model including fixed genetic groups } \\
$\mathrm{np}=20 \mathrm{P}=1 / 6$ & $5.66(0.029)$ & +0.27 & 14.1 & 14.1 & $5.09(0.037)$ & +0.54 & 15.4 & 68.7 \\
$\mathrm{np}=100 \mathrm{P}=1 / 6$ & $6.90(0.022)$ & +0.05 & 2.7 & 2.7 & $6.67(0.023)$ & +0.11 & 3.0 & 17.6 \\
$\mathrm{np}=20 \mathrm{P}=1 / 2$ & $2.75(0.013)$ & +0.35 & 14.1 & 14.1 & $2.36(0.020)$ & +0.75 & 15.4 & 68.7 \\
\hline
\end{tabular}

$\mathrm{R}$ and $\hat{\mathrm{R}}$ are the true and predicted selection responses obtained by Monte-Carlo simulation: expectations are derived from 2500 replicates. Empirical standard deviation of the expectation of $\mathrm{R}$ is given in brackets. $\mathrm{MSE}_{5-6}$ and $\mathrm{MSE}_{1-6}$ are the mean square errors of prediction of genetic differences between the two youngest cohorts and between the two extreme cohorts, respectively. 
Table II. Criteria derived from the genetic evaluation model, for varying number (np) of progeny per sire, for a trait of heritability $\mathrm{h}^{2}=0.20$, with an annual genetic trend $\Delta \mathrm{G}=0.2 \sigma_{\mathrm{a}}$ and a sire selection rate $\mathrm{P}=1 / 6$.

\begin{tabular}{|c|c|c|c|c|c|c|c|c|}
\hline & \multicolumn{4}{|c|}{ Reference sire design } & \multicolumn{4}{|c|}{ Repeater sire design } \\
\hline & $\mathrm{R}$ & $(\hat{\mathrm{R}}-\mathrm{R}) / \mathrm{R}$ & $\mathrm{MSE}_{5-6}$ & $\mathrm{MSE}_{1-6}$ & $\mathrm{R}$ & $(\hat{\mathrm{R}}-\mathrm{R}) / \mathrm{R}$ & $\mathrm{MSE}_{5-6}$ & $\mathrm{MSE}_{1-6}$ \\
\hline & \multicolumn{8}{|c|}{ Statistical model ignoring genetic groups } \\
\hline $\mathrm{np}=20$ & $3.47(0.034)$ & -0.04 & 2.4 & 17.1 & $3.30(0.035)$ & -0.02 & 3.0 & 22.0 \\
\hline \multirow[t]{2}{*}{$\mathrm{np}=100$} & $4.57(0.026)$ & -0.04 & 1.5 & 7.6 & $4.22(0.026)$ & -0.00 & 1.9 & 20.0 \\
\hline & \multicolumn{8}{|c|}{ Statistical model including random genetic groups } \\
\hline $\mathrm{np}=20$ & $3.43(0.037)$ & +0.24 & 7.2 & 10.6 & $3.25(0.036)$ & +0.03 & 3.8 & 21.9 \\
\hline \multirow[t]{2}{*}{$\mathrm{np}=100$} & $4.62(0.027)$ & +0.06 & 2.4 & 2.8 & $4.23(0.026)$ & +0.01 & 2.0 & 18.8 \\
\hline & \multicolumn{8}{|c|}{ Statistical model including fixed genetic groups } \\
\hline $\mathrm{np}=20$ & $3.13(0.039)$ & +0.71 & 15.4 & 15.4 & $2.68(0.051)$ & +1.21 & 14.0 & 64.5 \\
\hline $\mathrm{np}=100$ & $4.60(0.027)$ & +0.10 & 2.9 & 2.9 & $4.28(0.032)$ & +0.26 & 3.8 & 20.4 \\
\hline
\end{tabular}

$\mathrm{R}$ and $\hat{\mathrm{R}}$ are the true and predicted selection responses obtained by Monte-Carlo simulation: expectations are derived from 1000 replicates. Empirical standard deviation of the expectation of $\mathrm{R}$ is given in brackets. $\mathrm{MSE}_{5-6}$ and $\mathrm{MSE}_{1-6}$ are the mean square errors of prediction of genetic differences between the two youngest cohorts and between the two extreme cohorts, respectively. 
Table III. Number of sires selected across six years of evaluation in a repeater sire design, for a trait $\left(\mathrm{h}^{2}=0.4\right)$ with an annual genetic trend $\Delta \mathrm{G}=0.2 \sigma_{\mathrm{a}}$ (see text for a complete description of selection procedure).

\begin{tabular}{|c|c|c|c|c|c|c|}
\hline Year of evaluation & 1 & 2 & 3 & 4 & 5 & 6 \\
\hline \multicolumn{7}{|c|}{ Statistical model ignoring genetic groups } \\
\hline $\mathrm{np}=20 \quad \mathrm{P}=10 / 60$ & $1.6(1.0)$ & $1.7(1.0)$ & $1.7(1.0)$ & $1.7(1.0)$ & $1.7(1.0)$ & $1.7(1.0)$ \\
\hline $\mathrm{np}=100 \mathrm{P}=10 / 60$ & $1.5(1.0)$ & $1.6(1.1)$ & $1.6(1.1)$ & $1.6(1.1)$ & $1.7(1.1)$ & $1.8(1.1)$ \\
\hline$n p=20 \quad P=30 / 60$ & $4.9(1.4)$ & $5.0(1.5)$ & $5.0(1.5)$ & $5.0(1.5)$ & $5.0(1.5)$ & $5.1(1.4)$ \\
\hline \multicolumn{7}{|c|}{ Statistical model including random genetic groups } \\
\hline $\mathrm{np}=20 \quad \mathrm{P}=10 / 60$ & $1.6(1.1)$ & $1.7(1.1)$ & $1.7(1.1)$ & $1.7(1.1)$ & $1.7(1.2)$ & $1.7(1.2)$ \\
\hline $\mathrm{np}=100 \mathrm{P}=10 / 60$ & $1.4(1.1)$ & 1.6( & $1.6(1.1)$ & 1.7 & $1.7(1.1)$ & $2.0(1.2)$ \\
\hline $\mathrm{np}=20 \quad \mathrm{P}=30 / 60$ & $4.8(1.6)$ & $5.0(1.6)$ & $5.0(1.7)$ & $5.0(1.7)$ & $5.0(1.7)$ & $5.2(1.6)$ \\
\hline \multicolumn{7}{|c|}{ Statistical model including fixed genetic groups } \\
\hline $\mathrm{np}=20 \quad \mathrm{P}=10 / 60$ & $1.0(1.6)$ & $1.0(1.3)$ & $1.1(1.4)$ & $1.5(1.5)$ & $2.1(1.7)$ & $3.3(2.6)$ \\
\hline $\mathrm{np}=100 \mathrm{P}=10 / 60$ & $0.6(0.9)$ & $0.9(1.0)$ & $1.2(1.1)$ & $1.6(1.2)$ & $2.3(1.4)$ & $3.4(1.8)$ \\
\hline $\mathrm{np}=20 \quad \mathrm{P}=30 / 60$ & $3.0(3.1)$ & $3.6(2.9)$ & $4.3(2.7)$ & $5.5(2.6)$ & $6.5(3.0)$ & $7.1(3.3)$ \\
\hline
\end{tabular}

across years that was very close to the one obtained under an evaluation ignoring groups.

To further explain these results, Table IV presents the expectation of the true selection response as well as the distribution of sires selected across years under ideal conditions. The first ideal condition was a selection on true breeding values. By this way, the maximal true selection response and the optimal distribution of sires across years were determined. As expected, the optimal distribution was close to the distribution observed for a design with a high number of progeny recorded per sire $(n p=100)$ and with EBV accounting for fixed genetic groups. This design (Tab. I) gave $91 \%$ of the maximal selection response. Because the distribution of sires selected across year was only close to the optimal distribution when fitting fixed genetic groups, it might have been expected that the highest true selection response would always be obtained for that model. But it was only true with a high accuracy of EBV (np = 100). With a low accuracy of EBV $(n p=20)$, the increase of the prediction error variance counterbalanced the unbiased estimation of genetic groups: the standard deviations of the number of sires selected across years were strongly increased, indicating more errors in the ranking of sires across groups, although the average number of sires selected in each year was closest to the optimal distribution. An intuitive explanation of this result can be given by considering 
Table IV. True selection response (R) under optimal selection cases and number of sires selected across six years in the case of a selection process $(\mathrm{P}=1 / 6$ and $\mathrm{np}=20)$ in a repeater sire design, for a trait $\left(\mathrm{h}^{2}=0.4\right)$ with an annual genetic trend $\Delta \mathrm{G}=0.2 \sigma_{\mathrm{a}}$.

\begin{tabular}{ccccccc}
\hline Year of evaluation & 1 & 2 & 3 & 4 & 5 & 6 \\
\hline \multicolumn{7}{c}{ Selection on true breeding values } \\
$\mathrm{R}=7.30(0.019)$ & $0.5(0.7)$ & $0.8(0.8)$ & $1.2(1.0)$ & $1.7(1.1)$ & $2.4(1.3)$ & $3.4(1.4)$ \\
\hline Selection on EBV predicted without any “year of evaluation” fixed effects or genetic groups \\
$\mathrm{R}=6.24(0.026)$ & $0.6(0.8)$ & $0.9(0.9)$ & $1.3(1.0)$ & $1.8(1.0)$ & $2.3(1.2)$ & $3.1(1.4)$ \\
\hline Selection on EBV predicted without any “year of evaluation” but with random genetic groups \\
$\mathrm{R}=6.27$ (0.026) & $0.5(0.7)$ & $0.7(0.9)$ & $1.1(1.0)$ & $1.7(1.3)$ & $2.5(1.3)$ & $3.5(1.6)$ \\
\hline Selection on $\mathbf{E B V}$ predicted without any “year of evaluation” but with fixed genetic groups \\
$\mathrm{R}=6.27$ (0.026) & $0.4(0.7)$ & $0.6(0.8)$ & $1.1(1.0)$ & $1.7(1.3)$ & $2.5(1.4)$ & $3.7(1.6)$ \\
\hline
\end{tabular}


the case where there are no different genetic subpopulations in reality. This case was already presented in Section 2.3 to clarify the fact that selection response is always overestimated by including genetic groups in the evaluation. In that case, animals belonging to the overestimated groups will be chosen to the detriment of animals belonging to the underestimated groups and, hence, selection response can be lower than the one ignoring genetic groups for a given sample. Under repeated sampling, the estimates of genetic groups will be ranked in different orders because their expectations are null and, hence, the average distribution of sires selected across years will be optimal, although the average true selection response may be lower than the one derived under a model ignoring genetic groups.

\subsection{Effect of the confounding between the sire's year of birth and the year of evaluation}

The second ideal condition studied (Tab. IV) was a selection on EBV predicted when ignoring the effect of the year of evaluation. Let's recall that no real effects of the year of evaluation were simulated in the data and, consequently, the model ignoring the effects of years of sire evaluation was the best. This ideal case was studied to make clear that the heart of the problem was the existence of some confounding between genetic groups and years of evaluation of sires. In this ideal case and whatever the modelling of genetic groups, the EBV took fully into account the genetic trend, because the confounding of sire's birth year and its year of evaluation was avoided by ignoring the estimation of the environmental effects of years of the sire evaluation. Otherwise, the genetic trend was mainly accounted for in the estimates of these fixed effects of year of the sire evaluation, when genetic groups were ignored or treated as random effects. Table $\mathrm{V}$ presents the average estimates (over 2500 replicates) of these fixed effects, in the case of a repeater sire design and an annual genetic trend of $0.2 \sigma_{\mathrm{a}}$ equal to 1.265 . When genetic groups were considered as a fixed effect in the model, estimates of the effects "year of evaluation" were close to zero since they were sampled from a normal distribution with a zero mean.

Under the model ignoring the effects of year of sire evaluation (Tab. IV), distribution of sires selected across years was closer to optimal and true selection response was higher than the true response achieved when fitting effects of year of evaluation (Tab. I). The selection response was similar whatever the treatment of genetic groups (no, random or fixed effect). It was also close to the predicted response which was only slightly overestimated $(+3 \%)$ under a model fitting fixed genetic groups and slightly underestimated $(-2 \%)$ under 
Table V. Average estimates of the fixed effects "year of evaluation" in a repeater sire design $(\mathrm{np}=20)$, for a trait $\left(\mathrm{h}^{2}=0.4\right)$ with an annual genetic trend $\Delta \mathrm{G}=0.2 \sigma_{\mathrm{a}}$.

\begin{tabular}{ccccccc}
\hline Year of evaluation & 1 & 2 & 3 & 4 & 5 & 6 \\
\hline \multicolumn{7}{c}{ Statistical model ignoring genetic groups } \\
& 0.059 & 0.582 & 1.192 & 1.813 & 2.474 & 3.093 \\
\hline \multicolumn{7}{c}{ Statistical model including random genetic groups } \\
0.140 & 0.608 & 1.192 & 1.815 & 2.467 & 3.084 \\
\hline \multicolumn{7}{c}{ Statistical model including fixed genetic groups } \\
0 & -0.009 & -0.029 & -0.033 & -0.025 & -0.023 \\
\hline
\end{tabular}

a model ignoring genetic groups. Consequently, there was no real difference between models when year effects were ignored. But, in real-life, there will be environmental differences between years, and solutions for the remaining effects of sire evaluation would be biased if these fixed effects were ignored in the model.

\subsection{Effect of the data design on the criteria for model comparison}

The reference sire design always had lower MSE between extreme cohorts $\left(\mathrm{MSE}_{1-6}\right)$ compared to the repeater sire design. $\mathrm{MSE}_{5-6}$ was also lower for the reference sire design when no genetic groups were included in the evaluation model, but it was higher for this design when groups were fitted in the model.

True selection response was always higher for the reference sire design than for the repeater sire design. When including fixed genetic groups, the overestimation of selection response decreased when the design was better connected, e.g. the reference versus the repeater sire designs. The opposite result was obtained when considering random genetic groups.

\subsection{Effect of the selection accuracy on the criteria for model comparison}

A higher selection accuracy (larger values for $\mathrm{h}^{2}$ or $\mathrm{np}$ ) corresponded to a lower overestimation of selection response when including groups in the evaluation model (Tabs. I and II). This reduction of response bias was more important for fixed group models than for random group models. 
The effect of the proportion selected (P) across sire cohorts was tested (Tab. I). Under a model with fixed genetic groups, the overestimation of selection response was strongly increased with selected proportion; from $54 \%$ to $75 \%$ for a repeater sire design (27\% to $35 \%$ for a reference sire design) for $\mathrm{P}$ varying from $1 / 6$ to $1 / 2$. This increase was smaller under a model with random genetic groups.

\subsection{Effect of genetic trend on the criteria for model comparison}

Whatever the situation considered, the genetic trend was correctly predicted when including fixed group effects whereas it strongly regressed towards zero under a model ignoring groups and under a model with random genetic groups (Tab. VI). By this means, Monte-Carlo simulation confirmed that including fixed groups leads to unbiased prediction of genetic trend. This solution was proposed by Henderson [4] in his selection model for the type of selection that he called the "L'u selection". The strong regression towards zero of the prediction of genetic trend is due to the partial confounding between the fixed effects "year of evaluation" and the genetic effects of the birth year of sires, i.e. the genetic trend [9].

Comparing true selection responses under a genetic evaluation with or without genetic groups for a trait with $\mathrm{h}^{2}=0.4$ (Tab. VI) gave better responses to selection when ignoring genetic groups (or when treating them as a random effect) for $\Delta \mathrm{G}$ up to $0.2 \sigma_{\mathrm{a}}$. However, a better response was observed by including fixed genetic groups when $\Delta \mathrm{G}$ reached $0.3 \sigma_{\mathrm{a}}$. The selection bias in the models with fixed genetic groups decreased when the genetic difference between groups increased.

When comparing the MSE of the difference between two consecutive cohorts (numbered 5 and 6 in the tables), MSE under the model without genetic groups became greater than MSE under the model with genetic groups only when the genetic trend reached half a genetic standard deviation (unpublished results). Thus, the increase in error variance by including groups was too important to counterbalance the bias correction in the comparison of the genetic level of two consecutive cohorts. When the MSE of the difference between extreme cohorts $\left(\mathrm{MSE}_{1-6}\right)$ were compared, the model including fixed groups was preferred for genetic trends over $0.2 \sigma_{\mathrm{a}}$. This result was in agreement with the choice operated on the basis of the true selection responses. Below $0.2 \sigma_{\mathrm{a}}$, the model without genetic groups was preferred whatever the comparison criterion. 
Table VI. Selection bias induced by a between group selection process $(\mathrm{P}=1 / 6)$ in a repeater sire design $(n p=20)$ and MSE of sire evaluation for a trait $\left(h^{2}=0.40\right)$ with an annual genetic trend $\Delta \mathrm{G}$.

\begin{tabular}{|c|c|c|c|c|}
\hline True $\Delta \mathrm{G}$ & $0 \sigma \mathrm{a}$ & $0.1 \sigma \mathrm{a}$ & $0.2 \sigma \mathrm{a}$ & $0.3 \sigma \mathrm{a}$ \\
\hline \multicolumn{5}{|c|}{ Statistical model ignoring genetic groups } \\
\hline Predicted $\Delta \mathrm{G}$ & $0.000 \sigma \mathrm{a}$ & $0.000 \sigma \mathrm{a}$ & $0.003 \sigma \mathrm{a}$ & $0.004 \sigma \mathrm{a}$ \\
\hline $\mathrm{R}^{\mathrm{a}}$ & $5.40(1.26)$ & $5.40(1.30)$ & $5.39(1.38)$ & $5.47(1.55)$ \\
\hline$\hat{\mathrm{R}}^{\mathrm{b}}$ & $5.35(0.91)$ & $5.35(0.91)$ & $5.34(0.89)$ & $5.36(0.91)$ \\
\hline$(\hat{R}-\mathrm{R}) / \mathrm{R}$ & -0.01 & -0.01 & -0.01 & -0.02 \\
\hline $\mathrm{MSE}_{5-6}$ & 3.8 & 4.2 & 5.1 & 7.2 \\
\hline $\mathrm{MSE}_{1-6}$ & 3.9 & 13.8 & 42.6 & 91.8 \\
\hline \multicolumn{5}{|c|}{ Statistical model including random genetic groups } \\
\hline Predicted $\Delta \mathrm{G}$ & $-0.000 \sigma \mathrm{a}$ & $0.003 \sigma \mathrm{a}$ & $0.009 \sigma \mathrm{a}$ & $0.013 \sigma \mathrm{a}$ \\
\hline $\mathrm{R}^{\mathrm{a}}$ & $5.35(1.26)$ & $5.34(1.30)$ & $5.37(1.41)$ & $5.49(1.57)$ \\
\hline$\hat{\mathrm{R}}^{\mathrm{b}}$ & $5.45(0.95)$ & $5.45(0.94)$ & $5.46(0.94)$ & $5.49(0.96)$ \\
\hline$(\hat{R}-\mathrm{R}) / \mathrm{R}$ & +0.02 & +0.02 & +0.02 & +0.00 \\
\hline $\mathrm{MSE}_{5-6}$ & 4.9 & 5.2 & 5.9 & 7.9 \\
\hline $\mathrm{MSE}_{1-6}$ & 4.9 & 14.1 & 41.1 & 87.5 \\
\hline \multicolumn{5}{|c|}{ Statistical model including fixed genetic groups } \\
\hline Predicted $\Delta \mathrm{G}$ & $-0.000 \sigma \mathrm{a}$ & $0.100 \sigma \mathrm{a}$ & $0.200 \sigma \mathrm{a}$ & $0.300 \sigma \mathrm{a}$ \\
\hline $\mathrm{R}^{\mathrm{a}}$ & $4.30(1.60)$ & $4.52(1.68)$ & $5.09(1.86)$ & $5.99(2.04)$ \\
\hline$\hat{\mathrm{R}}^{\mathrm{b}}$ & $7.27(1.73)$ & $7.39(1.81)$ & $7.82(2.14)$ & $8.38(2.45)$ \\
\hline$(\hat{R}-\mathrm{R}) / \mathrm{R}$ & +0.69 & +0.64 & +0.54 & +0.40 \\
\hline $\mathrm{PEV}_{5-6}$ & 15.4 & 15.4 & 15.4 & 15.4 \\
\hline $\mathrm{PEV}_{1-6}$ & 68.7 & 68.7 & 68.7 & 68.7 \\
\hline
\end{tabular}

${ }^{\mathrm{a} b} \mathrm{R}$ and $\hat{\mathrm{R}}$ are the true and predicted selection responses obtained by Monte-Carlo simulation: expectation and standard deviation (in brackets) are derived from 1000 replicates, except for $\Delta \mathrm{G}=0.2 \sigma_{\mathrm{a}}$ (2500 replicates).

\section{DISCUSSION}

All criteria (selection bias, true selection response, mean squared error of the estimation of the difference between genetic groups) used for the choice of the genetic evaluation model converged towards the same conclusions:

(1) The more connected the design, the higher the selection response, whatever the model of evaluation; 
(2) whatever the data design, a model of genetic evaluation without groups is preferred to a model with genetic groups in terms of selection response when the genetic trend is in the range of likely values in animal breeding programs (0 to $20 \%$ of genetic standard deviation).

In cattle breeding programs, a maximal annual genetic trend of 0.2 genetic standard deviation is expected [9]. In such a case, including fixed genetic groups leads to a large overestimation of the predicted selection response, a smaller true selection response and a larger MSE of the difference between consecutive groups, when there is not enough information to get accurate reliabilities of sires. Including random genetic groups is not better in terms of maximisation of selection responses and minimisation of MSE between consecutive cohorts; it can only minimise MSE between extreme cohorts under highly connected designs.

In the above examples concerning planned connection in designs for beef cattle, including groups in a sire evaluation model to account for genetic trend is not a satisfying solution in terms of selection response because it leads to a very large increase in variance of prediction error of genetic differences. Hence, unbiasedness does not lead to faster genetic progress when the information is not sufficient. Last but not least, selection response will always be overestimated when considering fixed genetic groups and, consequently, the assessment of breeding program alternatives may be erroneous. It appears that the evaluation model and the data design should rather aim for a gain in accuracy of evaluation rather than to pursue the more a theoretical property of unbiasedness of the evaluation.

Including fixed groups of unknown ancestors in the pedigree of animals to be evaluated has become a frequent mean all over the world for genetic evaluation that accounts for possible genetic differences (mean and variance) in the base population [1]. Apart from the migration of animals from a genetically different nucleus into the population under current evaluation, differences among the base due to age are likely to be smaller than those considered in this simulation study. Consequently, the question arises of the overestimation of true selection responses and the evolution of mean square error of prediction under models that fit fixed genetic groups for base animals of different ages. The results may depend mainly on the accuracy of the evaluation and, to a certain extent, on the selection process, i.e. whether selection is between groups or within-group. In brief, the inclusion of genetic groups should be considered only with a large number of animals per group, high genetic links between groups and high accuracy of selection $\left(\mathrm{h}^{2}\right.$ and information available) and, above all, in the case of a priori large genetic differences between 
subpopulations of base animals. Said in another way by Kennedy and Moxley [8], "the need for grouping is greatest with high semen exchange, and with low semen exchange, grouping of sires will be counterproductive unless real differences between genetic groups are relatively large".

\section{REFERENCES}

[1] Alfonso L., Estany J., An expression of mixed animal model equations to account for different means and variances in the base population, Genet. Sel. Evol. 31 (1999) 105-113.

[2] Foulley J.L., Schaeffer L.R., Wilton J.W., Progeny group size in an organized progeny test program of AI beef sires using reference sires, Can. J. Anim. Sci. 63 (1983) 17-26.

[3] Foulley J.L., Hanocq E., Boichard D., A criterion for measuring the degree of connectedness in linear models of genetic evaluation, Genet. Sel. Evol. 24 (1992) 315-330.

[4] Henderson C.R., Sire evaluation and genetic trends, in: Proc. Anim. Breed. Genet. Symp, in Honor of Dr. Jay L. Lush, Am. Soc. Anim. Sci. and Am. Dairy Sci. Assoc., Champaign, IL, USA, 1973.

[5] Henderson C.R., Best linear unbiased estimation and prediction under a selection model, Biometrics 31 (1975) 423-435.

[6] Henderson C.R., Comparison of alternative sire evaluation methods, J. Anim Sci. 41 (1975) 760-771.

[7] Kennedy B.W., Bias and mean square error from ignoring genetic groups in mixed model sire evaluation, J. Dairy Sci. 64 (1981) 689-697.

[8] Kennedy B.W., Moxley J.E., Comparison of genetic group and relationship methods for mixed model sire evaluation, J. Dairy Sci. 58 (1975) 1507.

[9] Laloë D., Phocas F., A proposal of criteria of robustness analysis in genetic evaluation, Livest. Prod. Sci. 80 (2003) 241-256.

[10] Phocas F., Colleau J.J., Ménissier F., Expected efficiency of selection for growth in a French beef cattle breeding scheme. II. Prediction of asymptotic genetic gain in an heterogeneous population, Genet. Sel. Evol. 27 (1995) 171-188.

[11] Pollak E.J., Quaas R.L., Definition of group effects in sire evaluation models, J. Dairy Sci. 66 (1983) 1503-1509.

[12] Robinson G.K., Group effects and computing strategies for models for estimating breeding values, J. Dairy Sci. 69 (1986) 3106-3111.

[13] Westell R.A., Quaas R.L., Van Vleck L.D., Genetic Groups in an animal model, J. Dairy Sci. 71 (1988) 1310-1318. 